\title{
Correlations between Arteriograph-Derived Augmentation Index and Disease Activity in Acromegaly Patients.
}

\author{
Seçkin Dereli ${ }^{1}$, Canan Ersoy ${ }^{2}$, Ali Aydınlar ${ }^{3}$ \\ ${ }^{1}$ Ordu University Faculty of Medicine, Deparment of Cardiology, Ordu, Turkey \\ ${ }^{2}$ Uludag University, Faculty of Medicine, Department of Internal Medicine, Division of Endocrinology and Metabolism, Bursa, \\ Turkey. \\ ${ }^{3}$ Uludag University, Faculty of Medicine, Department of Cardiology, Bursa, Turkey.
}

Received: 07 July 2020, Accepted: 14 November 2020, Published online: 31 December 2020

(C) Ordu University Institute of Health Sciences, Turkey, 2020

\begin{abstract}
Objective: The high prevalence of cardiovascular diseases in acromegaly patients attracted attention to the association between the growth hormone and cardiovascular system. New markers such as the augmentation index (AIx) and central aortic pressure (CAP) have recently been introduced to the clinical practice for a variety of diagnostic and monitoring purposes in predicting arterial stiffness and associated endothelial dysfunction. In this present study, we aimed to determine the condition of the vascular system using the noninvasive arterial stiffness marker; AIx measured using by the Arteriograph device and to evaluate the relationship between these markers and the disease activity in acromegaly patients.

Methods: This was a cross-sectional study. The study included 53 acromegaly patients and 20 individuals age and sex matched as controls. The arterial stiffness was evaluated to estimate the AIx and CAP with a low-frequency suprasystolic waveform analysis in the occluded brachial artery, performed by using a Cardio Scope II Arteriograph device, which was adapted from a standard sphygmomanometer.

Results: The mean augmentation index was statistically significantly different between the three groups, being $98 \%$ in the active acromegaly (AA) group, $92 \%$ in controlled acromegaly (CA) and $79.5 \%$ in the control group ( $\mathrm{p}=0.001)$. In the acromegaly group, the results of the regression analysis indicated a strong correlation of the arterial stiffness parameters with $\mathrm{HbAlc}$ and the platelet counts, however, the levels of IGF-1 and GH were not correlated

Conclusion: It might be thought that AIx may have an important role in predicting the cardiovascular risk in acromegaly.

Key words: Augmentation index; disease activity; acromegaly; arterial stiffness;
\end{abstract}

Suggested Citation: Dereli S, Ersoy C, Aydinlar A. Correlations between Arteriograph-derived augmentation index and disease activity in acromegaly patients. Middle Black Sea Journal of Health Science, 2020; 6(3):376-384.

Address for correspondence/reprints:

Seçkin Dereli

DOI: $\quad 10.19127 / \mathrm{mbsjohs.764508}$

Telephone number: +90 (533) 3760984

ORCID-ID 0000-0003-0090-3835

E-mail: drseckindereli@gmail.com 


\section{Introduction}

Acromegaly is a syndrome characterised by the secretion of growth hormone $(\mathrm{GH})$ and insulin-like growth factor (IGF-1) (Colao et al. 2001). The longterm exposure of tissues to excessive $\mathrm{GH}$ in acromegaly leads to increases in the cardiovascular disease-associated morbidity and mortality by two- to three-folds. The coronary heart disease and hypertension are the most important causes of increased mortality and morbidity in acromegaly patients (Bengtsson et al. 1988).

The development of vascular abnormalities has been demonstrated in acromegaly patients even in the absence of cardiovascular risk factors. Studies have shown that the abnormal GH secretion may be associated with an increased risk of atherosclerosis in acromegaly (Bengtsson et al. 1988). In the liver, the GH stimulates the production of IGF-1, which mediates the GH activity in the peripheral tissues (Sowers, 1997). GH and IGF-1 have significant effects on the cardiovascular system (Celermajer et al. 1992, Neunteufl et al. 2000). In acromegaly, GH assume its effects on the endothelium via the endothelial IGF-1 receptors directly or via the lipid metabolism indirectly (Tan et al. 1997). It affects the IGF-1's regulating function on the vascular tonus, attenuating the vascular contractility and triggering the endothelial dysfunction (Walsh et al. 1996).

Endothelial dysfunction and arterial stiffness in acromegaly patients have received a considerable attention in recent years (Smith et al. 2003). Several parameters have recently been introduced to assess arterial stiffness and have proven to be the independent predictors of untoward cardiovascular outcomes. (Nichols, 2005, Laurent et al. 2006) Pulse wave analysis has been used in most studies to investigate arterial stiffness in acromegaly. (Smith, et al. 2003; Sakai et al. 2008; Paisley et al. 2011). Augmentation index (AIx) is the amplification ratio of the central arterial waveform pressure attributed to the reflected pulse pressure. Central AIx has been reported to be closely associated with several risk factors for atherosclerosis and future cardiovascular events (London et al. 2001; Izzo, 2004; Weber, et al. 2004) and in these studies AIx had measured by using applanation tonometry. However, in Yaron et al. (2016) study, there were no significant differences in Alx between acromegaly and control subjects. AIx can be estimated from the brachial artery waveform as peripheral AIx and can provide information on arterial stiffness, as it is closely related to the central aortic AIx (Manisty and Hughes, 2013).

While arterial stiffness can be measured in many different ways, few studies have focused on arterial stiffness in acromegaly using peripheral arteries (Matsuda, Kawate et al. 2013). The aim of our study was to investigate the brachial artery AIx as an arterial stiffness parameter by using arteriograph device in a group of acromegaly patients with active acromegaly (AA) controlled acromegaly (CA) compared with control group. Also, we want to demonstrate the correlation between the endothelial dysfunction and the disease activity in acromegaly patients.

\section{Methods}

\section{Study population}

The study included acromegaly patients presented to the endocrinology outpatient clinic of the internal medicine department of Uludag University between May 2015 and August 2015. In order to prevent any interferences with the cardiovascular assessments, the patients were excluded from the study if they had a previously known structural heart disease (congenital/rheumatic heart valve disease, arrhythmia, or heart failure), coronary artery disease, a diagnosis of cancer, renal or hepatic failure, an acute or chronic infectious disease, a diagnosis of autoimmune disease or had a history of undergoing a major surgery or trauma in the recent 3 months.

It was planned to include acromegaly patients who were followed up in the endocrinology outpatient clinic. During the endocrinology outpatient clinic application, the patients were asked to be included in the study and were evaluated. After the preliminary evaluation, 21 patients were excluded from the study for the reasons stated above. A total of 53 patients were included in the study.

A control group comprised the individuals with diabetes or hypertension at ages between 20 and 80 years, in whom the diagnosis of acromegaly was excluded. The individuals in the control group were matched with those in the patient group in terms of age and gender.

\section{Assessment of Disease Activity}

The diagnosis of acromegaly was made with the presence of classical clinical features, higher serum IGF-1 levels by age, and GH concentrations of $1 \mu \mathrm{g} / \mathrm{L}$ $(\mathrm{ng} / \mathrm{ml})$ or higher after a 75 -gram OGTT.

The patients with acromegaly were assigned to two groups as active acromegaly (AA) and controlled acromegaly (CA) according to the disease activity and the consensus criteria for cure of acromegaly (Melmed et al. 2018) The patients with GH levels reduced below $1 \mu \mathrm{g} / \mathrm{L}(\mathrm{ng} / \mathrm{ml})$ and the patients achieving IGF-1 levels within the age-adjusted limits 
were included in the cured acromegaly group. A total of 32 patients in the study were included in this group meeting the criteria for cure. In the active acromegaly group, a total of 21 patients, who did not meet the criteria for cure were included. Of these 21 patients, four were newly diagnosed acromegaly patients and 10 were the patients who did not meet the cure criteria despite the ongoing hormone suppressor therapy.

All patients were questioned about the duration of symptoms before clinical diagnosis, the treatments they received, any systemic involvement in association with acromegaly, and the co-morbid systemic diseases. The following independent risk factors for cardiovascular diseases were evaluated, including diabetes mellitus (DM), hypertension (HT), hyperlipidemia, obesity, and smoking.

The height $(\mathrm{cm})$ and body weight $(\mathrm{kg})$ of the patients were recorded and their body mass indices (BMI) $\mathrm{kg} / \mathrm{m} 2$ were calculated. The results of the laboratory tests performed at the routine outpatient control visits were recorded including the fasting plasma glucose, total cholesterol, triglycerides, HDL, LDL, GH, IGF-1, HbA1c, and hemoglobin levels and the platelet and leukocyte counts.

\section{Measurement of Arterial Stiffness}

Patients who were included in the study at the time of the endocrinology outpatient clinic admission were evaluated. At the same time, cardiac evaluation was performed. Our aim was to measure the central SBP, DBP and brachial AIx as the primary output variables. The patients had a rest for 15 minutes, staying away from external stimuli after the control visit in the endocrinology outpatient clinic. In this study, arterial stiffness was assessed with the blood pressure (BP) and the central blood pressure in the brachial artery in a non-invasive manner using the Cardio Scope II device. After completing the pressure measurements with the specified device, the cuff was inflated to a level above the current systolic pressure (at least $35 \mathrm{mmHg}$ ). This way, occlusion of the brachial artery was accomplished, stopping the blood flow during the measurement according to the procedure protocol (only 8-20 seconds, with a mean of 8 seconds). In this very specific condition (stopflow condition), a membrane develops in the brachial artery at the level of the upper margin of the inflated cuff, where the blood flow was stopped. When the central pressure changes and early (direct P1) and late (reflected backwards; P2) systolic and diastolic waves reach the site of occlusion, they became detectable like the pulsating blood beating the membrane. Like a transferring medium, the upper arm tissue transfers the small but sensible changes, caused by the pressure of the flowing fluid, to the cuff along the skin and the border of the cuff, allowing them being generalized. The high-resolution pressure sensors of the arteriography are capable of detecting these small and weak changes in the pressure. Two components of the measured pulse wave, the pulse wave from the heart and the reflection wave at the periphery, were analyzed. The data were processed with a specific software developed for this purpose and the central SBP, DBP, and AIx values were recorded.

\section{Statistical analyses}

It was calculated that at least 38 patients should be included in the study for AIx $90 \%$ in acromegaly patients with an alpha value of 0.05 and a strength of $80 \%$ in the power analysis program. The SPSS 22.0 statistical package program (SPSS Inc., Illinois, USA) was used to perform all data analyses. The Kolmogorov-Smirnov test was used to analyze the distribution pattern. Normally distributed numerical variables were presented as mean \pm standard deviation, whereas the ones not normally distributed were presented as median and interquartile range. Categorical variables were presented as number and percent $(\%)$. Basal characteristics were classified according to predefined subgroups and the parametric variables of the three independent groups were evaluated using analysis of variance with/without Bonferroni. The categorical variables were evaluated using the appropriate Chi-square test. The KruskalWallis $\mathrm{H}$ test with/without Bonferroni correction was used while examining the three groups in the analysis of variables that do not fit the normal distribution. The Spearman rank test was performed to define the correlation of disease activity and arterial stiffness. The relationship coefficient was expressed as " $r$ " and the significance value was expressed as "p".

\section{Results}

Based on the previously defined disease activity criteria; 21 active acromegaly (AA) and 32 controlled cured acromegaly (CA) patients were included in the study. Table 1 presents the comparison of the patient and control groups in terms of their demographic and basic clinical features and in terms of the risk factors for cardiovascular diseases. There were no statistically significant differences in gender or age between the control group and the two patient groups. Body mass index was higher in both AA and CA groups compared to the control group when all acromegaly patients were compared to the control group and also when AA and CA groups were 
individually compared to the control group $(\mathrm{p}=$ 0.001).

There were no significant differences in terms of treatment duration or the history of surgery or radiotherapy between the active acromegaly and cure acromegaly groups. The medication use was higher in the AA group compared to the CA group $(p=0.001)$. DM rate was statistically significantly higher in the AA group compared to the CA and control groups $(\mathrm{p}=0.001)$. HT was found to be at similar rates in the CA and AA groups; however, the rates of HT in these groups were higher compared to the control group. The rates of HL and smoking were similar in all three groups. Obesity was observed at similar rates in the $\mathrm{CA}$ and AA groups; but these rates were higher than that of the control group.

Table 2 lists the biochemical parameters compared between the groups. The levels of growth hormone, IGF-1, glucose, and HbA1c were significantly higher in the AA group compared to the CA and control groups $(p=0.001)$. The levels of HDL, LDL, and haemoglobin; and the WBC counts were not different among the groups. TG levels were higher in the control group $(\mathrm{p}=0.001)$. The platelet count was higher in the AA and CA groups compared to the control group $(p=0.001)$.

Arterial stiffness parameters measured with the arteriograph devices were presented in Table 3. In our study, the arterial stiffness parameters including AIx, central SBP, central DBP; and SBP and DBP were significantly higher in the AA group compared to both CA and control groups $(\mathrm{p}=0.001)$. The AIx, central SBP and DBP were significantly higher in the CA group compared to the control group $(p=0.001)$.

Correlation analysis of AIx are presented in Table 4. In acromegaly patients, there was a positive and strong correlation of AIx with the HbA1c levels and the platelet count [respectively ( $\mathrm{r}: 0.699, \mathrm{p}<0.01$ ) $(\mathrm{r}: 0.796, \mathrm{p}<0.01)]$. The correlation analysis showed a positive and strong correlation of AIx with APG, $\mathrm{HbA1c}$, and BMI in the control group [respectively $(\mathrm{r}: 0.535, \mathrm{p}<0.01) \quad(\mathrm{r}: 0.660, \mathrm{p}<0.01) \quad(\mathrm{r}: 0.796$, $\mathrm{p}<0.01)]$.

Table 1. Clinical characteristics of acromegaly patients and healthy control

\begin{tabular}{|c|c|c|c|}
\hline & $\mathbf{A A}(\mathbf{n}=21)$ & CA $(n=32)$ & Control(n=20) \\
\hline Age, years & $45.5 \pm 14.2$ & $48.7 \pm 12.7$ & $47.5 \pm 10.3$ \\
\hline Gender, $\mathbf{f} / \mathrm{m}$ & $9 / 12$ & $13 / 17$ & $9 / 11$ \\
\hline BMI, $\mathrm{kg} / \mathrm{m}^{2}$ & $29,3 \pm 4.1 \dagger$ & $29,4 \pm 4.5^{*}$ & $24,5 \pm 3.6$ \\
\hline Surgery, $\mathrm{n}$ & 20 & 31 & - \\
\hline \multicolumn{4}{|l|}{ Drug use, $n$} \\
\hline Somatostatin analogs & 11 & 12 & - \\
\hline Dopamine agonist & 3 & 1 & - \\
\hline GH receptor ant. & 2 & 1 & - \\
\hline TOTAL, n (\%) & $16(\% 76) \div$ & $14(\% 46)$ & - \\
\hline Radiotherapy, n (\%) & $4 / 21$ & $6 / 32$ & \\
\hline Treatment duration & $7.8 \pm 5.0$ & $7.4 \pm 3.9$ & \\
\hline DM, n (\%) & $8(\% 38)+\hbar$ & $8(\% 25)$ & $5(\% 25)$ \\
\hline HT, n (\%) & $11(\% 53) \dagger$ & $16(\% 51) *$ & $5(\% 25)$ \\
\hline HL, n (\%) & $4(\% 19)$ & $6(\% 20)$ & $3(\% 15)$ \\
\hline Obezite, n (\%) & $7(\% 33)+$ & $11(\% 34) *$ & $1(\% 5)$ \\
\hline Sigara, n (\%) & $5(\% 19)$ & $7(\% 21)$ & $4(\% 20)$ \\
\hline
\end{tabular}

AA: active acromegaly; CA: Controlled acromegaly, F: female; M: male; BMI: body mass index; GH; growth hormone; DM: diabetes mellitus; HL: hyperlipidemia; HT: hypertension;

$* \mathrm{P}<0.01$ for controls versus KA

$\dagger \mathrm{P}<0.01$ for controls versus AA

$\$ \mathrm{P}<0.01$ for KA versus AA 
Table 2. Biochemical measurements in acromegaly and control groups

\begin{tabular}{|c|c|c|c|}
\hline & $\mathbf{A A}(\mathbf{n}=21)$ & CA $(n=32)$ & Control $(n=20)$ \\
\hline GH, ng/ml & $2.37(1.1-40.0)^{\dagger \dagger}$ & $0.46(0.060 .99)$ & $0.51(0.431 .63)$ \\
\hline IGF-1, ng/ml & $265(91-982)^{\dagger}$ & $206(49-417)^{*}$ & $157(121-203)$ \\
\hline Glucose, $\mathrm{mg} / \mathrm{dl}$ & $120.4 \pm 23.7^{\dagger *}$ & $103.2 \pm 40.5$ & $99.6 \pm 8.7$ \\
\hline HDL, mg/dl & $43.0 \pm 10.7$ & $42.5 \pm 9.5$ & $43.9 \pm 6.7$ \\
\hline LDL, mg/dl & $115(63-272)$ & $114(51-292)$ & 112(79-192) \\
\hline TG, mg/dl & $136(64-441)^{\dagger *}$ & $115(26-272)^{*}$ & 187(103-303) \\
\hline Hemoglobin, mg/dl & $13.3 \pm 1.44$ & $13.6 \pm 1.3$ & $13.5 \pm 1.1$ \\
\hline WBC, $10^{3} / \mathbf{u l}$ & $7757 \pm 1400$ & $8128 \pm 1209$ & $7705 \pm 1059$ \\
\hline PLT, $10^{3} /$ ul & $286900 \pm 64100 \dagger$ & $297000 \pm 48200^{*}$ & $235000 \pm 48200$ \\
\hline HBA1c, mg/dl & $6.1(5.1-12.5)^{\dagger \dagger}$ & $5.6(5.0-9.5)$ & $5.5(5.0-9.6)$ \\
\hline
\end{tabular}

AA: active acromegaly; CA: Controlled acromegaly, GH: growth hormone; IGF-1: insülin like growht factor-1; HDL: high density lipoprotein; LDL: low density lipoprotein; TG: triglyceride; WBC: White blood cell; PLT: platelet; HBA1c: hemoglobin A1c;

$* \mathrm{P}<0.01$ for controls versus $\mathrm{KA}$

${ }^{\dagger} \mathrm{P}<0.01$ for controls versus AA

$\ddagger \mathrm{P}<0.01$ for KA versus AA

Table 3. Arterial stiffness parameters acromegaly and control groups

\begin{tabular}{lccc}
\hline & AA $(\mathbf{n}=21)$ & CA $(\mathbf{n}=32)$ & Control(n=20) \\
\hline AIx, \% & $98(74-145)^{\dagger \ddagger}$ & $92(75-126)^{*}$ & $79.5(69-96)$ \\
\hline SBP, $\mathbf{~ m m} / \mathbf{H g}$ & $133.8 \pm 10.2^{\dagger \ddagger}$ & $129.9 \pm 9.5^{*}$ & $125.5 \pm 11.3$ \\
\hline DBP, $\mathbf{~ m m} / \mathbf{H g}$ & $84.4 \pm 8.2^{\dagger \ddagger}$ & $83.2 \pm 8.7^{*}$ & $77.3 \pm 10.7$ \\
\hline Central SBP, $\mathbf{~ m m} / \mathbf{H g}$ & $123.5 \pm 10.1^{\dagger \ddagger}$ & $119.6 \pm 9.2^{*}$ & $115.5 \pm 11.1$ \\
\hline Central DBP, $\mathbf{~ m m} / \mathbf{H g}$ & $88.5 \pm 9.5^{\dagger \ddagger}$ & $84.8 \pm 7.6^{*}$ & $78.5 \pm 10.8$ \\
\hline
\end{tabular}

AA: active acromegaly; CA: Controlled acromegaly; AIx: augmentation index; SBP: Systolic blood pressure; DKB: Diastolic blood pressure

${ }^{*} \mathrm{P}<0.01$ for controls versus KA

$\dagger \mathrm{P}<0.01$ for controls versus AA

$\$ \mathrm{P}<0.01$ for KA versus AA

Tablo 4. Correlation analysis of Augmentation index

\begin{tabular}{lcccc}
\hline \multirow{2}{*}{ Parameters } & \multicolumn{2}{c}{ Acromegaly $(\mathbf{n}=\mathbf{5 3})$} & \multicolumn{2}{c}{ Control $(\mathbf{n}=\mathbf{2 0})$} \\
\cline { 2 - 5 } GH & $\mathrm{r}$ & $\mathrm{p}$ & \multicolumn{1}{c}{$\mathrm{p}$} & $\mathrm{p}$ \\
\hline IGF-1 & 0.067 & $\mathrm{p}>0.05$ & 0.148 & $\mathrm{p}>0.05$ \\
\hline Glucose & -0.331 & $\mathrm{p}>0.05$ & 0.074 & $\mathrm{p}>0.05$ \\
\hline TG & 0.410 & $\mathrm{p}>0.05$ & 0.535 & $\mathrm{p}<0.01$ \\
\hline PLT & -0.077 & $\mathrm{p}>0.05$ & -0.066 & $\mathrm{p}>0.05$ \\
\hline HBA1c & 0.796 & $\mathrm{p}<0.01$ & -0.22 & $\mathrm{p}>0.05$ \\
\hline BMI & 0.699 & $\mathrm{p}<0.01$ & 0.660 & $\mathrm{p}<0.01$ \\
\hline
\end{tabular}

GH: growth hormone; IGF-1: insülin like growht factor-1; TG: triglyceride; PLT: platelet; HBA1c: hemoglobin A1c; BMI: body mass index

\section{Discussion}

Our study is the first, in which the augmentation index measured with the cardioscope device was used to determine the arterial stiffness in predicting the cardiovascular risk in acromegaly patients. AIx and the central aortic blood pressure were significantly increased in acromegaly patients compared to the controls. Arterial stiffness markers were found to be associated with the disease activity; however, there was not a significant correlation between the biochemical parameters.
High levels of GH and IGF-1 in the patients cause structural and functional changes in the heart and vascular system. These specific changes developing in the heart and the vascular bed in the absence of hypertension or diabetes mellitus occur starting from the early stages of the disease; however, the clinical symptoms become manifest in years (Morvan et al. 1991). This leads the cardiovascular involvement to progress without being noticed. Several studies have shown that cardiac dysfunction is alleviated with the curative treatment given in the early stages of cardiac 
involvement (Parolin, Dassie et al. 2018). However, the regression of the vascular bed alterations was not satisfactory. For example, it was reported that the increase in the IMT persisted due to the high insulin levels in the patients including the cured ones (Colao et al. 2001). Endothelial dysfunction and arterial stiffness increase as a result of early pathophysiological and structural changes in the cardiovascular system.

In acromegaly patients, a method or a diagnostic test that can identify the cardiovascular involvement in the early asymptomatic phase will allow for making the diagnosis of cardiovascular diseases at earlier stages and taking necessary preventive measures before disease progression. Non-invasive methods, such as pulse wave velocity, flow-mediated vasodilation, cardio-ankle vascular index, and ambulatory arterial stiffness index, have been used in a variety of studies to identify the subclinical structural changes, leading to the development of arterial stiffness in acromegaly. (Davies and Struthers 2003, Baykan et al. 2009, Matsuda et al. 2013). Among these non-invasive methods, AIx receives attention as it is easy and fast to measure, therefore, AIx was used to detect arterial stiffness in different clinical conditions (Fischer-Rasokat et al. 2009, Eguchi et al. 2016, García-Ortiz et al. 2018). Aortic pressure wave comprises a forward propagating wave caused by the left ventricular ejection and the retrograde waves reflected back from the periphery. Therefore, the augmentation of the aortic pressure waveform is a manifestation of wave reflection. To make it clear, the augmentation pressure or the augmentation index (AIx) can be expressed as a percentage of the pulse pressure. Peripheral augmentation index (PAIx) is defined as the ratio of the systolic peaks and is considered as a measure of the arterial functions (Rosenbaum et al. 2013, Eguchi et al. 2016). A high PAIx causes an increased systemic vascular resistance resulting in an increased systolic load. Therefore, it indicates that the myocardial hypertrophy is increased (Izzo, 2014). Several studies have associated PAIx with cardiovascular morbidity and mortality and demonstrated that it predicts the untoward cardiac events independently. (Fischer-Rasokat et al. 2009). In this study, we selected to investigate PAIx as an indicator of arterial stiffness because our aim was to use an easily applicable practical method. Measurements were made using a Cardio Scope II device. This non-invasive and sphygmomanometerbased device is easy to use and provides highly precise and reproducible measurements of several parameters, including the (aortic) systolic and diastolic blood pressures, AIx, and the pulse waveform (Lin et al. 2012). The Cardio Scope uses the common upper arm cuff with conventional oscillometry in a fast, continuous, and simple manner without the need for additional training and without the requirement for additional sensors. In addition, it provides signal quality indices and data on the pulse rate variability.

In our study, one of our aims was to evaluate the correlation between the arterial stiffness parameters and disease markers. But the correlation analysis did not reveal any association of AIx with either GH or IGF-1. This might have occurred due to the low number of study patients and the heterogeneity of the parameters such as the duration of the disease. It may be suggested that the most important reason was the difficulties in performing OGGT, leading to an unsatisfactory reflection of the disease activity by IGF-1 and GH levels used in the diagnosis and follow-up of the disease. It is recommended that only IGF-1 should be measured in the patients receiving growth hormone receptor antagonists (Trainer et al. 2000). A multi-centre study has reported that $30 \%$ of the lowest GH levels after OGTT were inconsistent with the actual diagnosis (Pokrajac et al. 2007). There are several studies reporting that OGTT may not be needed if the levels of IGF-1 and GH are significantly higher. On the other hand, there are other studies indicating that the use of OGTT may not be appropriate to determine the disease activity in patients receiving medical treatment (Carmichael et al. 2009). Our study also included patients receiving radiotherapy. It has been reported that GH levels could be normal despite high levels of IGF-1 after radiotherapy, as radiotherapy ensures more stable levels of GH instead of a pulsatile secretion (Van Gelder et al. 2002).

The DM frequency was similar in the patient and control groups of our study; however, the mean $\mathrm{HbA1c}$ levels were higher in the AA group. Lee Shin et al. (2016) and Cavero-Redondo et al. (2018) demonstrated in their studies that there was a positive association between arterial stiffness and $\mathrm{HbA} 1 \mathrm{c}$ levels. Similar to the previous studies, the correlation analysis in our study revealed a positive correlation between $\mathrm{HbA} 1 \mathrm{c}$ and AIx in both the patient group and the control group.

\section{Limitations}

We recognise the limitations of our study. First, this is a single-centre study conducted with a relatively small number of patients. Further multicentre studies with a larger patient population will be beneficial. The participants of our study did not have 
overt cardiovascular diseases, however, some of them received antihypertensive, antidiabetic or statin medications with a potential to affect the measurement of AIx. The presence of a coronary heart disease could not be excluded completely as no invasive stress tests or angiographies have been performed; however, the presence of ischaemia was excluded by the clinical, electrocardiographic, or echocardiographic findings. A final possible limitation can be that an imaging of the arterial system of the upper extremities was not performed in the patients prior to the measurements, and that the potential presence of arterial stenoses might have affected the measurements.

\section{Conclusion}

In this study, AIx has been demonstrated as a parameter associated with arterial stiffness in predicting the cardiovascular risk in patients with acromegaly. As the use of Cardio Scope is simple and swift, it might be thought that AIx measured by using this device can be easy and economical in the prediction of arterial stiffness in patients with acromegaly, helping physicians identify the high-risk patients. Further prospective studies are required to investigate AIx in predicting arterial stiffness in a variety of clinical conditions in acromegaly as this parameter is practical to measure with a low-cost.

Acknowledgment: I thank the Dr Guven Ozkaya for their expertise and assistance throughout statistical analysis of our study.

Ethics Committee Approval: It was approved for this study by the Uludağ University Faculty of Medicine Clinical Research Ethics Committee on 0906-2015 with decision number 2015-12 / 27.

Peer-review: Externally peer-reviewed.

Author Contributions: Concept -S.D., C.E.,A.A.; Design -C.E.; Supervision -A.A.; Resource -C.E.; Materials -S.D.; Data collection \&/or processing S.D.; Analysis \&/or interpretation -S.D.; Literature search -S.D., C.E.; Writing -S.D.; Critical review C.E., A.A.

Conflict of Interest: No conflict of interest was declared by the author.

Financial Disclosure: The author declared that this study hasn't received no financial support.

\section{References}

Baykan M, Erem C, Gedikli O, Hacihasanoglu A, Erdogan T, Kocak M, et al. Impairment in flowmediated vasodilatation of the brachial artery in acromegaly. Med Princ Pract. 2009;18(3):228232. doi:10.1159/000204355

Bengtsson BA, Edén S, Ernest I, Odén A, Sjögren B. Epidemiology and long-term survival in acromegaly. A study of 166 cases diagnosed between 1955 and 1984. Acta Med Scand. 1988;223(4):327-335. doi:10.1111/j.09546820.1988.tb15881.x

Carmichael JD, Bonert VS, Mirocha JM, Melmed S. The utility of oral glucose tolerance testing for diagnosis and assessment of treatment outcomes in 166 patients with acromegaly. Clin Endocrinol Metab. 2009;94(2):523-527. doi:10.1210/jc.20081371

Cavero-Redondo I, Martínez-Vizcaíno V, ÁlvarezBueno C, Recio-Rodríguez JI, Gómez-Marcos MÁ, García-Ortiz L. Relationship between glycaemic levels and arterial stiffness in nondiabetic adults. Relación entre los valores de glucemia y la rigidez arterial en adultos no diabéticos. Med Clin (Barc). 2018;150(2):56-60. doi:10.1016/j.medcli.2017.06.072.

Celermajer DS, Sorensen KE, Gooch VM, Spiegelhalter DJ, Miller BMed OI, Sullivan Fracp ID. et al. Non-invasive detection of endothelial dysfunction in children and adults at risk of atherosclerosis. Lancet. 1992;340(8828):11111115. doi:10.1016/0140-6736(92)93147-f.

Colao A, Marzullo P, Di Somma C, Lombardi G. Growth hormone and the heart. Clin Endocrinol (Oxf). 2001;54(2):137-154. doi:10.1046/j.13652265.2001.01218.x.

Colao A, Spiezia S, Cerbone G, Pivonella R, Marzullo P, Ferone D. et al. Increased arterial intima-media thickness by B-M mode echodoppler ultrasonography in acromegaly. Clin Endocrinol (Oxf). 2001;54(4):515-524. doi:10.1046/j.1365-2265.2001.01256.x.

Davies JI, Struthers AD. Pulse wave analysis and pulse wave velocity: a critical review of their strengths and weaknesses. J Hypertens. 2003;21(3):463-472. doi:10.1097/00004872200303000-00004.

Eguchi K, Hoshide S, Miyashita H, Nagasaka S, Kario K. Why the radial augmentation index is low in patients with diabetes: The J-HOP study. Atherosclerosis. 2016;246:338-343. doi:10.1016/j.atherosclerosis.2016.01.034. 
Fischer-Rasokat U, Brenck F, Zeiher AM, Spyridopoulos I. Radial augmentation index unmasks premature coronary artery disease in younger males. Blood Press Monit. 2009;14(2):59-67. doi:10.1097/MBP.0b013e32832941ce.

García-Ortiz L, Recio-Rodríguez JI, Agudo-Conde C, Mandereuelo-Fernandez JA, Patino-Alonso MC, Cabo-Laso A, et al. Noninvasive validation of central and peripheral augmentation index estimated by a novel wrist-worn tonometer. J Hypertens. 2018;36(11):2204-2214. doi:10.1097/HJH.0000000000001806

Izzo JL Jr. Arterial stiffness and the systolic hypertension syndrome. Curr Opin Cardiol. 2004;19(4):341-352.

doi:10.1097/01.hco.0000126581.89648.10.

Izzo JL Jr. Brachial vs. central systolic pressure and pulse wave transmission indicators: a critical analysis. Am J Hypertens. 2014;27(12):14331442. doi:10.1093/ajh/hpu135.

Laurent S, Cockcroft J, Van Bortel L, BoutouyrieP, Giannattasio C, Hayoz D, et al. Expert consensus document on arterial stiffness: methodological issues and clinical applications. Eur Heart J. 2006;27(21):2588-2605. doi:10.1093/eurheartj/ehl254.

Lee YH, Shin MH, Choi JS, Rhee JA, Nam HS, Jeong $\mathrm{SK}$, et al. HbA1c is significantly associated with arterial stiffness but not with carotid atherosclerosis in a community-based population without type 2 diabetes: The Dong-gu study. Atherosclerosis. 2016;247:1-6. doi:10.1016/j.atherosclerosis.2016.01.032.

Lin AC, Lowe A, Sidhu K, Harrison W, Ruygrok P, Stewart R. Evaluation of a novel sphygmomanometer, which estimates central aortic blood pressure from analysis of brachial artery suprasystolic pressure waves. J Hypertens. 2012;30(9):1743-1750. doi:10.1097/HJH.0b013e3283567b94.

London GM, Blacher J, Pannier B, Guérin AP, Marchais SJ, Safar ME. Arterial wave reflections and survival in end-stage renal failure. Hypertension. 2001;38(3):434-438. doi:10.1161/01.hyp.38.3.434.

Manisty $\mathrm{CH}$, Hughes AD. Meta-analysis of the comparative effects of different classes of antihypertensive agents on brachial and central systolic blood pressure, and augmentation index. $\mathrm{Br} \quad \mathrm{J}$ Clin Pharmacol. 2013;75(1):79-92. doi:10.1111/j.1365-2125.2012.04342.x.
Matsuda Y, Kawate H, Matsuzaki C, Sakamoto R, Abe I, Shibue K. et al. Reduced arterial stiffness in patients with acromegaly: non-invasive assessment by the cardio-ankle vascular index (CAVI). Endocr J. 2013;60(1):29-36. doi:10.1507/endocrj.ej12-0189.

Melmed S, Bronstein MD, Chanson P, Klibanski A, Casanueva FF, Wass JAH, et al. A Consensus Statement on acromegaly therapeutic outcomes. Nat Rev Endocrinol. 2018 Sep;14(9):552-561. doi: 10.1038/s41574-018-0058-5.

Morvan D, Komajda M, Grimaldi A, Turpin G, Grosgogeat Y. Cardiac hypertrophy and function in asymptomatic acromegaly. Eur Heart $\mathrm{J}$. 1991;12(6):666-672..

Neunteufl T, Heher S, Katzenschlager R, Wölfl G, Kostner K, Maurer G, Weidinger F. Late prognostic value of flow-mediated dilation in the brachial artery of patients with chest pain. Am J Cardiol. 2000;86(2):207-210. doi:10.1016/s00029149(00)00857-2.

Nichols WW. Clinical measurement of arterial stiffness obtained from noninvasive pressure waveforms. Am J Hypertens. 2005;18(1 Pt 2):3S10S. doi:10.1016/j.amjhyper.2004.10.009.

Paisley AN, Banerjee M, Rezai M, Schofield RE, Balakrishnannair S, Herbert A, et al. Changes in arterial stiffness but not carotid intimal thickness in acromegaly. J Clin Endocrinol Metab. 2011;96(5):1486-1492. doi:10.1210/jc.20102225.

Parolin M, Dassie F, Martini C, Mioni R, Russo L, Fallo F, et al. Preclinical markers of atherosclerosis in acromegaly: a systematic review and meta-analysis. Pituitary. 2018;21(6):653-662. doi:10.1007/s11102-0180911-5.

Pokrajac A, Wark G, Ellis AR, Wear J, Wieringa GE, Trainer PJ. Variation in GH and IGF-I assays limits the applicability of international consensus criteria to local practice. Clin Endocrinol (Oxf). 2007;67(1):65-70. doi:10.1111/j.13652265.2007.02836.x.

Rosenbaum D, Giral P, Chapman J, Rached FH, Kahn $\mathrm{JF}$, Bruckert E, et al. Radial augmentation index is a surrogate marker of atherosclerotic burden in a primary prevention cohort. Atherosclerosis. 2013;231(2):436-441. doi:10.1016/j.atherosclerosis.2013.10.004. 
Sakai H, Tsuchiya K, Nakayama C, Iwashima F, Izumiyama $\mathrm{H}$, Doi $\mathbf{M}$, et al. Improvement of endothelial dysfunction in acromegaly after transsphenoidal surgery. Endocr J. 2008;55(5):853-859. doi:10.1507/endocrj.k07e125.

Smith JC, Lane H, Davies N, Evans LM, Cockcroft J, Scanlon MF, et al. The effects of depot long-acting somatostatin analog on central aortic pressure and arterial stiffness in acromegaly. J Clin Endocrinol Metab. 2003;88(6):2556-2561. doi:10.1210/jc.2002-021746.

Sowers JR. Insulin and insulin-like growth factor in normal and pathological cardiovascular physiology. Hypertension. 1997;29(3):691-699. doi:10.1161/01.hyp.29.3.691.

Tan KC, Shiu SW, Janus ED, Lam KS. LDL subfractions in acromegaly: relation to growth hormone and insulin-like growth factor-I. Atherosclerosis. 1997;129(1):59-65. doi:10.1016/s0021-9150(96)06015-7.

Trainer PJ, Drake WM, Katznelson L, Freda PU, Herman-Bonert V, van der Lely AJ, et al. Treatment of acromegaly with the growth hormone-receptor antagonist pegvisomant. N Engl J Med 2000; 342:1171-1177 DOI: 10.1056/NEJM200004203421604.

Van Gelder IC, Hagens VE, Bosker HA, Kingma JH, Kamp O, Kingma T, et al. A comparison of rate control and rhythm control in patients with recurrent persistent atrial fibrillation. $\mathrm{N}$ Engl $\mathrm{J}$ Med. 2002;347(23):1834-1840. doi:10.1056/NEJMoa021375.

Walsh MF, Barazi M, Pete G, Muniyappa R, Dunbar JC, Sowers JR. Insulin-like growth factor I diminishes in vivo and in vitro vascular contractility: role of vascular nitric oxide. Endocrinology. $\quad 1996 ; 137(5): 1798-1803$. doi:10.1210/endo.137.5.8612517.

Weber T, Auer J, O'Rourke MF, Kvas E, Lassnig E, Berent $\mathrm{R}$ et al. Arterial stiffness, wave reflections, and the risk of coronary artery disease. Circulation. 2004;109(2):184-189. doi:10.1161/01.CIR.0000105767.94169.E3.

Yaron M, Izkhakov E, Sack J, Azzam I, Osher E, Tordjman K, et al. Arterial properties in acromegaly: relation to disease activity and associated cardiovascular risk factors. Pituitary. 2016;19(3):322-331. doi:10.1007/s11102-0160710-9. 